九州大学学術情報リポジトリ

Kyushu University Institutional Repository

\title{
CLOSURE SYSTEMS AND CLOSURE OPERATIONS IN DEDEKIND CATEGORIES
}

Kawahara, Yasuo

Department of Informatics, Kyushu University

Tanaka, Kakuj i

Department of Informatics, Kyushu University

https://doi.org/10.5109/18996

出版情報: Bulletin of informatics and cybernetics. 40, pp.89-99, 2008-12. Research Association of Statistical Sciences

バージョン:

権利関係 : 


\section{CLOSURE SYSTEMS AND CLOSURE OPERATIONS IN DEDEKIND CATEGORIES}

by

Yasuo KAWAHARA and Kakuji TANAKA

Reprinted from the Bulletin of Informatics and Cybernetics Research Association of Statistical Sciences, Vol.40

FUKUOKA, JAPAN

2008 


\title{
CLOSURE SYSTEMS AND CLOSURE OPERATIONS IN DEDEKIND CATEGORIES
}

\author{
By
}

\author{
Yasuo KAWAHARA* and Kakuji TANAKA ${ }^{\dagger}$
}

\begin{abstract}
The concepts of closure systems and closure operations in lattice theory are basic and applied to many fields in mathematics and theoretical computer science. In this paper authors find out a suitable definition of closure systems in Dedekind categories, and thereby give an equivalence proof for closure systems and closure operations in Dedekind categories.
\end{abstract}

Key Words and Phrases: Closure system, Closure operation, Dedekind category

\section{Introduction}

Closure systems and closure operations in complete ordered sets are one of the basic concepts in mathematics and theoretical computer science. For example, the closure systems and closure operations are not only used in algebraic systems, graph theory, a definition of topological spaces and formal concept analysis ,introduced by Ganter and Wille (1999) and Ishida, Honda and Kawahara (2008), but also related with Galois connections. Tarski (1941) and Freyd and Scedrov (1990) extensively developed the mathematical framework for relational methodology. Schmidt and Ströhlein (1993) and Schmidt (2006) proposed that the framework is applied to computer science.

Now we will briefly review the fundamentals on infima, closure systems and closure operations in ordered sets. Let $(X, \leq)$ be an ordered set. For a subset $T$ of $X$ an element $a$ of $X$ is called the infimum (greatest lower bound) of $T$ if

$$
x \leq a \leftrightarrow \forall t \in T . x \leq t
$$

for all $x \in X$. The infimum does not always exist. The infimum of $T$ will be denoted by $\inf T$ (if it exists). An ordered set $(X, \leq)$ is complete if for all subsets $T$ of $X$ there exists the infimum of $T$. Closure systems and closure operations are defined in complete ordered sets.

Let $(X, \leq)$ be a complete ordered set. A subset $S$ of $X$ is a closure system if $T \subseteq S \operatorname{implies} \inf T \in S$. A mapping $f: X \rightarrow X$ is a closure operation if it is extensive $(x \leq f(x))$, idempotent $(f f(x)=f(x))$ and monotonic $(x \leq y$ implies $f(x) \leq f(y))$. The motivation of the paper arose from the following four fundamental exercises in a complete ordered set $(X, \leq)$ :

(i) The image $f(X)$ of a closure operation $f: X \rightarrow X$ is a closure system.

\footnotetext{
* Department of Informatics, Kyushu University, Fukuoka, 819-0395, Japan. kawahara@i.kyushuu.ac.jp

$\dagger$ Department of Informatics, Kyushu University
} 
(ii) If $S$ is a closure system of $X$, then the mapping $f_{S}: X \rightarrow X$ defined by $f_{S}(x)=$ $\inf S_{x}$ is a closure operation, where $S_{x}=\{t \in X \mid t \in S$ and $x \leq t\}$.

(iii) If $f: X \rightarrow X$ is a closure operation, then $f_{f(X)}=f$ holds.

(iv) If $S$ is a closure system of $X$, then $f_{S}(X)=S$ holds.

In this paper the authors aim to study closure systems and closure operations in Dedekind categories and to prove the above four statements (i), (ii), (iii) and (iv) with relational methodology. In general it may be so heavy to implement set theory or predicate logic on computers. However, in Bergharmmer and Neumann (2005), the Dedekind categories are algebraically defined by a few conditions and so their implementation would be much easier. The setting and definitions are eventually simple but the proofs of our main results (Theorem 4.4, 4.5 and 4.6) seem to be relatively technical. Also we will glance at point axioms and membership relations to supply spread around this subject. However the reader should remark that the statements and the proofs of the main results are independent of the point axioms ,introduced by Kawahara (2008), and membership relations introduced by Freyd and Scedrov (1990) and Ishida, Honda and Kawahara (2008).

The paper organised as follows. In Section 2 we recall some fundamentals on Dedekind categories which are our background. In Section 3 we mention three candidates of the definition of closure systems in Dedekind categories. In Section 4 we demonstrate the above statements (i), (ii), (iii) and (iv) in Dedekind categories.

\section{Dedekind Categories}

In this section we recall a notion of Dedekind categories, due to Mac Lane (1999) and Olivier and Serrato (1980), which is an abstraction of the category of sets and binary relations or the category of sets and fuzzy relations. Throughout this paper, a morphism $\alpha$ from an object $X$ into an object $Y$ in a Dedekind category (defined below) will be denoted by a half arrow $\alpha: X \rightarrow Y$, and the composition of a morphism $\alpha: X \rightarrow Y$ followed by a morphism $\beta: Y \rightarrow Z$ will be written as $\alpha \beta: X \rightarrow Z$. Also we will denote the identity morphism on $X$ as $\operatorname{id}_{X}$.

Definition 2.1. A Dedekind category $\mathcal{D}$ is a category satisfying the following four conditions:

DC1. [Complete Heyting Algebra] For all pairs of objects $X$ and $Y$ the hom-set $\mathcal{D}(X, Y)$ consisting of all morphisms of $X$ into $Y$ is a complete Heyting algebra with the least morphism $0_{X Y}$ and the greatest morphism $\nabla_{X Y}$. Its algebraic structure will be denoted by

$$
\mathcal{D}(X, Y)=\left(\mathcal{D}(X, Y), \sqsubseteq, \sqcup, \sqcap, \Rightarrow, 0_{X Y}, \nabla_{X Y}\right),
$$

where $\sqsubseteq, \sqcup, \sqcap$ and $\Rightarrow$ denote the inclusion order, the join, the meet and the relatively pseudo-complement of morphisms, respectively.

DC2. [Converse] There is given a converse operation $\sharp: \mathcal{D}(X, Y) \rightarrow \mathcal{D}(Y, X)$. That is, for all morphisms $\alpha, \alpha^{\prime}: X \rightarrow Y$ and $\beta: Y \rightarrow Z$, the converse laws hold: (a) $(\alpha \beta)^{\sharp}=\beta^{\sharp} \alpha^{\sharp}$, (b) $\left(\alpha^{\sharp}\right)^{\sharp}=\alpha$, (c) If $\alpha \sqsubseteq \alpha^{\prime}$, then $\alpha^{\sharp} \sqsubseteq \alpha^{\prime \sharp}$.

DC3. [Dedekind Formula] For all morphisms $\alpha: X \rightarrow Y, \beta: Y \rightarrow Z$ and $\gamma: X \rightarrow Z$ the Dedekind formula $\alpha \beta \sqcap \gamma \sqsubseteq \alpha\left(\beta \sqcap \alpha^{\sharp} \gamma\right)$ holds. 
DC4. [Residual Composition] For all morphisms $\alpha: X \rightarrow Y$ and $\beta: Y \rightarrow Z$ the residual composition $\alpha \ominus \beta: X \rightarrow Z$ is defined as a morphism such that $\gamma \sqsubseteq \alpha \ominus \beta$ if and only if $\alpha^{\sharp} \gamma \sqsubseteq \beta$ for all morphisms $\gamma: X \rightarrow Z$.

An object $I$ of a Dedekind category is called a (strict) unit if $0_{I I} \neq \mathrm{id}_{I}=\nabla_{I I}$ and $\nabla_{X I} \nabla_{I X}=\nabla_{X X}$ for all objects $X$. The unit $I$ plays a rôle as substitute for a singleton set.

In what follows, all statements are assumed to be those in a Dedekind category with a unit $I$ (unless otherwise stated) and the word relation is a synonym for morphism in the Dedekind category.

A relation $f: X \rightarrow Y$ is called a function, denoted by $f: X \rightarrow Y$, if it is univalent $\left(f^{\sharp} f \sqsubseteq \operatorname{id}_{Y}\right)$ and total $\left(\operatorname{id}_{X} \sqsubseteq f f^{\sharp}\right)$. The universal relation $\nabla_{X I}: X \rightarrow I$ and the identity relation $\operatorname{id}_{X}: X \rightarrow X$ are functions. Also it is trivial that $\nabla_{X I} \nabla_{I Y}=\nabla_{X Y}$ holds for all objects $X$ and $Y$. An $I$-point $x$ of $X$, denoted by $x \in X$, is a function $x: I \rightarrow X$. For a relation $\rho: I \rightarrow X$ the notation $x \in \rho$ will denote that $x$ is an $I$-point of $X$ with $x \sqsubseteq \rho$. The residual composition will be frequently used in the paper. For example, the supremum $\sup (\rho, \xi): V \rightarrow X$ is defined by

$$
\sup (\rho, \xi)=(\rho \ominus \xi) \sqcap\left[(\rho \ominus \xi) \ominus \xi^{\sharp}\right]
$$

for a pair of relations $\rho: V \rightarrow X$ and $\xi: X \rightarrow X$. Dually the infimum $\inf (\rho, \xi)$ is defined by $\inf (\rho, \xi)=\sup \left(\rho, \xi^{\sharp}\right)$.

The basic properties of Dedekind categories are listed in the following proposition. For the proof refer to Appendix in Kawahara (2007).

Proposition 2.2. Let $\alpha, \alpha^{\prime}: X \rightarrow Y, \beta, \beta^{\prime}: Y \rightarrow Z, \gamma: Z \rightarrow U, \delta: U \rightarrow Z$, $\rho: V \rightarrow X$ and $\xi: X \rightarrow X$ relations. Then the following holds.

(a) If $\alpha \sqsubseteq \alpha^{\prime}$ and $\beta \sqsubseteq \beta^{\prime}$ then $\alpha \beta \sqsubseteq \alpha^{\prime} \beta^{\prime}$,

(b) If $\alpha \sqsubseteq \alpha^{\prime}$ and $\beta \sqsubseteq \beta^{\prime}$ then $\alpha^{\prime} \ominus \beta \sqsubseteq \alpha \Theta \beta^{\prime}$,

(c) $\alpha\left(\sqcup_{j} \beta_{j}\right)=\sqcup_{j} \alpha \beta_{j}$ and $\left(\sqcup_{j} \alpha_{j}\right) \beta=\sqcup_{j} \alpha_{j} \beta$,

(d) If $\alpha$ and $\delta$ are univalent then $\alpha\left(\beta \sqcap \beta^{\prime}\right) \delta^{\sharp}=\alpha \beta \delta^{\sharp} \sqcap \alpha \beta^{\prime} \delta^{\sharp}$,

(e) If $\alpha$ is total, $\alpha^{\prime}$ is univalent and $\alpha \sqsubseteq \alpha^{\prime}$ then $\alpha=\alpha^{\prime}$,

(f) $\alpha \ominus(\beta \ominus \gamma)=\alpha \beta \ominus \gamma$ and $(\alpha \ominus \beta) \gamma \sqsubseteq \alpha \ominus \beta \gamma$,

(g) $\alpha \sqsubseteq(\alpha \ominus \beta) \ominus \beta^{\sharp}$,

(h) $\alpha \odot \beta=\left[(\alpha \odot \beta) \ominus \beta^{\sharp}\right] \ominus \beta$,

(i) If $\alpha$ is a function then $\alpha \ominus \beta=\alpha \beta$ and $\alpha(\beta \ominus \gamma)=\alpha \beta \ominus \gamma$,

(j) If $\beta$ is a function then $\alpha \beta \ominus \gamma=\alpha \ominus \beta \gamma$,

(k) If $\delta$ is a function then $(\alpha \ominus \beta) \delta^{\sharp}=\alpha \ominus \beta \delta^{\sharp}$, 
(l) $\sup \left(\rho, \xi^{\sharp}\right)=\sup \left(\rho \ominus \xi^{\sharp}, \xi\right)$,

(m) $\sup (\rho, \xi) \sqsubseteq \rho \ominus \xi \sqsubseteq \sup (\rho, \xi) \ominus \xi$,

(n) If $\xi \xi \sqsubseteq \xi$ and $\sup (\rho, \xi)$ is total then $\sup (\rho, \xi) \xi=\rho \ominus \xi$,

(o) If $f: W \rightarrow V$ is a function then $f \sup (\rho, \xi)=\sup (f \rho, \xi)$,

(p) If $\xi \sqcap \xi^{\sharp} \sqsubseteq \operatorname{id}_{X}$ then $\sup (\rho, \xi)$ is univalent.

An endorelation $\xi: X \neg X$ is called an order if it is reflexive (id $\mathrm{id}_{X} \sqsubseteq \xi$ ), transitive $(\xi \xi \sqsubseteq \xi)$ and antisymmetric $\left(\xi \sqcap \xi^{\sharp} \sqsubseteq \mathrm{id}_{X}\right)$. Note that $\xi$ is reflexive and transitive if and only if $\xi=\xi^{\sharp} \ominus \xi$.

\section{Closure Systems}

In this section we will study the basic feature for closure systems with respect to orders in Dedekind categories. First of all we will introduce so-called point axioms:

(PA1) For all objects $X$ the identity $\nabla_{I X}=\sqcup_{x \in X} x$ holds.

(PA2) For all relations $\rho: I \longrightarrow X$ the identity $\rho=\sqcup_{x \in \rho} x$ holds.

The first point axiom (PA1) holds in the category $\operatorname{Rel}_{L}($ Set $)$ of sets and $L$-relations as well as the category $\operatorname{Rel}(\mathrm{Set})$ of sets and binary relations. Of course the second point axiom (PA2) comprises (PA1). A Dedekind category $\mathcal{D}$ is said to be representable if it can be embedded in $\operatorname{Rel}(S e t)$, namely there is a faithful relator from $\mathcal{D}$ into $\operatorname{Rel}(\operatorname{Set})$. In a representable Dedekind category almost all statements valid in $\operatorname{Rel}(\operatorname{Set})$ hold. (PA2) is so strong as to hold only in representable Dedekind categories. (PA2) fails even in the Dedekind category of sets and fuzzy relations, because a lot of fuzzy relations from a singleton set into a set have no expression as a sum of points which are basically crisp.

Extending the notions of membership predicate $\epsilon$ and power sets in set theory one can define membership relations and power objects in Dedekind categories as follows: For an object $Y$ a relation $\ni_{Y}: \wp(Y) \rightarrow Y$ is called a membership relation if for all relations $\alpha: X \rightarrow Y$ there is a unique function $\alpha^{@}: X \rightarrow \wp(Y)$ such that $\alpha=\alpha^{@} \ni_{Y}$. Then the domain $\wp(Y)$ of $\ni_{Y}$ is called the power object of $Y$. The relation $\Xi_{X}=$ $\ni_{X} \ominus \ni_{X}^{\sharp}: \wp(X) \rightarrow \wp(X)$ is an abstraction of inclusion order (or containment) of subsets. $\Xi_{X}$ is clearly reflexive and transitive. Contrarily its antisymmetry need not hold. But the paper will concern only the definition and the reflexivity of $\Xi_{X}$.

Proposition 3.1. Let $\ni_{X}: \wp(X) \rightarrow X$ be a membership relation and $\rho, \nu: I \rightarrow X$ relations. Then $\rho \sqsubseteq \nu$ if and only if $\rho^{@} \sqsubseteq \nu^{@} \Xi_{X}^{\sharp}$.

Proof. First note that $\rho^{@} \Xi_{X}{ }^{\sharp}=\left(\ni_{X} \ominus \rho^{\sharp}\right)^{\sharp}$ always holds:

$$
\begin{aligned}
\rho^{@} \Xi_{X} & =\rho^{@}\left(\ni_{X} \ominus \ni_{X}\right)^{\sharp} & & \left\{\Xi_{X}=\ni_{X} \ominus \ni_{X}^{\sharp}\right\} \\
& =\left(\ni_{X} \odot \ni_{X} \rho^{\circledR \sharp}\right)^{\sharp} & & \left\{\rho^{@} \text { : function and } 2.2(\mathrm{k})\right\} \\
& =\left(\ni_{X} \odot \rho^{\sharp}\right)^{\sharp} . & & \left\{\rho^{@} \ni_{X}=\rho\right\}
\end{aligned}
$$


Assume $\rho^{@} \sqsubseteq \nu^{@} \Xi_{X}^{\sharp}$. Then it holds that

$$
\begin{aligned}
\rho & =\rho^{@} \ni_{X} \\
& \sqsubseteq \nu^{@} \Xi_{X} \ni_{X} \\
& \sqsubseteq \nu^{@} \ni_{Y} \quad\left\{\ni_{X} \Xi_{X} \sqsubseteq \ni_{X}^{\sharp}\right\} \\
& =\nu .
\end{aligned}
$$

Conversely assume $\rho \sqsubseteq \mu$. Then we have

$$
\begin{array}{rlrl}
\rho^{@} & \sqsubseteq \rho^{@} \Xi_{X} & \left\{\operatorname{id}_{\wp(X)} \sqsubseteq \Xi_{X}\right\} \\
& =\left(\ni_{X} \ominus \rho^{\sharp}\right)^{\sharp} & \\
& \sqsubseteq\left(\ni_{X} \ominus \nu^{\sharp}\right)^{\sharp} & \{\rho \sqsubseteq \nu \text { and } 2.2(\mathrm{~b})\} \\
& =\nu^{@} \Xi_{X} .
\end{array}
$$

Intuitively a closure system is simply a subset $C$ of a complete ordered set in which the infimum for all subsets of $C$ is in $C$. The following two propositions state three candidates (that is, the statements (a), (b) and (c) in Proposition 3.3) for relational definition of closure systems. The statement (a) may be a naive abstraction of closure systems in Dedekind categories. Firstly we will show that the statements (a) and (b) are equivalent under the assumption (PA1).

Proposition 3.2. Let $\xi: X \rightarrow X$ and $\nu: I \rightarrow X$ be relations. Under the assumption of the point axiom (PA1) the following two statements are equivalent.

(a) $\rho \sqsubseteq \nu$ implies $\inf (\rho, \xi) \sqsubseteq \nu$ for all relations $\rho: I \rightarrow X$.

(b) $\nabla_{I V} \mu \sqsubseteq \nu$ implies $\nabla_{I V} \inf (\mu, \xi) \sqsubseteq \nu$ for all relations $\mu: V \rightarrow X$.

Proof. (b) $\rightarrow$ (a) (b) is a generalisation of (a). That is, setting $V=I$ in (b) yields (a). (a) $\rightarrow$ (b) Assume $\nabla_{I V} \mu \sqsubseteq \nu$. Then for $x \in V$ it holds that $x \mu \sqsubseteq \nabla_{I V} \mu \sqsubseteq \nu$ and so $\inf (x \mu, \xi) \sqsubseteq \nu$ by (a). Hence we have

$$
\begin{aligned}
\nabla_{I V} \inf (\mu, \xi) & =\left(\sqcup_{x \in V} x\right) \inf (\mu, \xi) & & \{(\mathrm{PA} 1)\} \\
& =\sqcup_{x \in V} x \inf (\mu, \xi) & & \\
& =\sqcup_{x \in V} \inf (x \mu, \xi) & & \{x: \text { function and } 2.2(\mathrm{o})\} \\
& \sqsubseteq \nu . & & \{\inf (x \mu, \xi) \sqsubseteq \nu\}
\end{aligned}
$$

Applying the point axiom (PA2) and the membership relation the statement (a) is equivalent to (c) in addition to (b).

Proposition 3.3. Let $\xi: X \rightarrow X$ and $\nu: I \rightarrow X$ be relations. Under the assumptions of the point axiom (PA2) and the existence of membership relations the following three statements are equivalent.

(a) $\rho \sqsubseteq \nu$ implies $\inf (\rho, \xi) \sqsubseteq \nu$ for all relations $\rho: I \rightarrow X$,

(b) $\nabla_{I V} \mu \sqsubseteq \nu$ implies $\nabla_{I V} \inf (\mu, \xi) \sqsubseteq \nu$ for all relations $\mu: V \rightarrow X$, 
(c) $\nu^{@} \Xi_{X}^{\sharp} \inf \left(\ni_{X}, \xi\right) \sqsubseteq \nu$.

Proof. In the last Proposition 3.2 we have already seen that (a) and (b) are equivalent under (PA1).

$(\mathrm{a}) \rightarrow$ (c) We will use the point axiom (PA2) here. Let $s \in \nu^{@} \Xi_{X} \sharp$. Then $\left(s \ni_{X}\right)^{@}=$ $s \sqsubseteq \nu^{@} \Xi_{X}^{\sharp}$ and so $s \ni_{X} \sqsubseteq \nu$ by Proposition 3.1. Hence we have $\inf \left(s \ni_{X}, \xi\right) \sqsubseteq \nu$ by the hypothesis (a) and

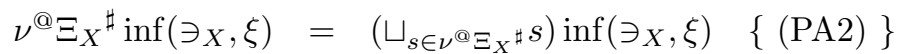

$$
\begin{aligned}
& =\sqcup_{s \in \nu^{\circledR} \Xi_{X}} s \inf \left(\ni_{X}, \xi\right)
\end{aligned}
$$

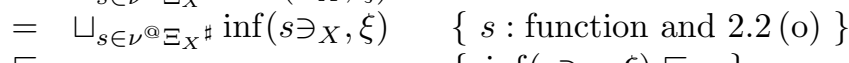

$$
\begin{aligned}
& \sqsubseteq \nu, \quad\left\{\inf \left(s \ni_{X}, \xi\right) \sqsubseteq \nu\right\}
\end{aligned}
$$

which proves (c).

(c) $\rightarrow$ (b) Assume $\nabla_{I V} \mu \sqsubseteq \nu$. (It is trivial that $\nabla_{I V} \mu \sqsubseteq \nu$ if and only if $\mu \sqsubseteq \nabla_{V I} \nu$.) Then $\mu \sqsubseteq \nabla_{V I} \mu$ and so $\mu^{@} \sqsubseteq\left(\nabla_{V I} \nu\right)^{@} \Xi_{X}^{\sharp}=\nabla_{V I} \nu^{@} \Xi_{X}^{\sharp}$ by Proposition 3.1. Hence we have

$$
\begin{aligned}
\inf (\mu, \xi) & =\mu^{@} \inf \left(\ni_{X}, \xi\right) & & \left\{\mu=\mu^{@} \ni_{X}\right\} \\
& \sqsubseteq \nabla_{V I} \nu^{@} \Xi_{X} \inf \left(\ni_{X}, \xi\right) & & \left\{\mu^{@} \sqsubseteq \nabla_{V I} \nu^{@} \Xi_{X} \sharp\right. \\
& \sqsubseteq \nabla_{V I} \nu . & & \{(\mathrm{c})\}
\end{aligned}
$$

This completes the proof.

Remark that implications $(\mathrm{b}) \rightarrow(\mathrm{a})$ and $(\mathrm{c}) \rightarrow(\mathrm{b})$ in the last Proposition 3.3 have been proved without point axioms. The statement (c) looks like a simple definition of closure systems in Dedekind categories. However it is too strong, because when we may adopt it as the definition of closure systems we encounter some difficulty to show Theorem 4.4 below, one of main results in the paper. On the other hand the statement (a) is so weak that the proof of Theorem 4.6 (b) fails. Originally category theory is an algebraic system without elements and so we prefer to establish theory of closure systems without the point axioms. Hence closure systems in Dedekind categories are formally defined by using the statement (b) in 3.3. For more detailed discussion the reader should refer Remark 1 and 2 after Theorem 4.4 and 4.6, respectively.

Definition 3.4. Let $\xi: X \rightarrow X$ be a relation. A relation $\nu: I \rightarrow X$ is a closure system (with respect to $\xi$ ) if $\nabla_{I V} \mu \sqsubseteq \nu$ implies $\nabla_{I V} \inf (\mu, \xi) \sqsubseteq \nu$ for all relations $\mu: V \rightarrow X$.

In what follows the point axioms (PA1) and (PA2) as well as the completeness of Heyting algebras in Definition 2.1 (DC1) will not formally assumed.

\section{Closure Operations}

In this section we will describe the definition of closure operations in Dedekind categories and demonstrate the equivalence between closure systems and closure operations in a relational fashion. Usually closure operations are extensive, idempotent and monotonic endofunctions. As the definition is quite transparent and universal, it is natural to reach the following definition.

Definition 4.1. Let $\xi: X \rightarrow X$ be a relation. A function $f: X \rightarrow X$ is a closure operation (with respect to $\xi$ ) if $f \sqsubseteq \xi, f f=f$ and $\xi \sqsubseteq f \xi f^{\sharp}$. 
To discuss the equivalence of closure systems and closure operations we need that involved infima are functions.

Definition 4.2. A relation $\xi: X \rightarrow X$ is called complete if $\sup (\mu, \xi): V \rightarrow X$ is total for all relations $\mu: V \rightarrow X$.

A relation $\xi$ is complete if and only if for all relations $\mu: V \rightarrow X$ the infimum $\inf (\mu, \xi): V \rightarrow X$ is total. Recall $\inf (\mu, \xi)=\sup \left(\mu \ominus \xi^{\sharp}, \xi\right)$.

The following proposition shows that the completeness of endorelations reflects the ordinary completeness defined for all subsets of the universe of discourse.

Proposition 4.3. Let $\ni_{X}: \wp(X) \rightarrow X$ be a membership relation. A relation $\xi: X \rightarrow X$ is complete if and only if $\sup \left(\ni_{X}, \xi\right)$ is total.

Proof. It is trivial that the completeness of $\xi$ implies the totality of $\sup \left(\ni_{X}, \xi\right)$. Now assume that $\sup \left(\ni_{X}, \xi\right)$ is total. Then for all relations $\mu: V \rightarrow X$ we have

$$
\begin{aligned}
& \sup (\mu, \xi)=\sup \left(\mu^{@} \ni_{X}, \xi\right) \quad\left\{\mu=\mu^{@} \ni_{X}\right\} \\
& =\mu^{@} \sup \left(\ni_{X}, \xi\right), \quad\left\{\mu^{@}: \text { function }\right\}
\end{aligned}
$$

which proves that $\sup (\mu, \xi)$ is total.

As stated in the introduction the image of a closure operation induces a closure system. This indicates the following theorem in the relational setting.

TheOREm 4.4. Let $\xi: X \rightarrow X$ be a complete order. If $f: X \rightarrow X$ is a closure operation, then the relation $\nu_{f}=\nabla_{I X} f: I \rightarrow X$ is a closure system.

Proof. Assume $\mu \sqsubseteq \nabla_{V I} \nabla_{I X} f=\nabla_{V X} f$. To see $\inf (\mu, \xi) \sqsubseteq \nabla_{V X} f$ it suffices to show $\inf (\mu, \xi) \sqsubseteq \inf (\mu, \xi) f$. First note that $\mu \sqsubseteq \nabla_{V X} f$ iff $\mu=\mu f^{\sharp} f=\mu f$. Then it holds that

$$
\begin{aligned}
\inf (\mu, \xi) f & \sqsubseteq\left(\mu \ominus \xi^{\sharp}\right) f & & \\
& \sqsubseteq \mu \ominus \xi^{\sharp} f & & \{2.2(\mathrm{f})\} \\
& \sqsubseteq \mu \ominus f \xi^{\sharp} & & \left\{\xi^{\sharp} \sqsubseteq f \xi^{\sharp} f^{\sharp}\right\} \\
& =\mu f \ominus \xi^{\sharp} & & \{2.2(\mathrm{j})\} \\
& =\mu \ominus \xi^{\sharp} & & \{\mu f=\mu\} \\
& =\inf (\mu, \xi) \xi^{\sharp}, & & \{\xi: \text { complete and } 2.2(\mathrm{n})\}
\end{aligned}
$$

and so

$$
\begin{aligned}
\inf (\mu, \xi) f & =\inf (\mu, \xi) f \sqcap \inf (\mu, \xi) \xi^{\sharp} & & \left\{\inf (\mu, \xi) f \sqsubseteq \inf (\mu, \xi) \xi^{\sharp}\right\} \\
& =\inf (\mu, \xi)\left(f \sqcap \xi^{\sharp}\right) & & \{\inf (\mu, \xi): \text { function }\} \\
& \sqsubseteq \inf (\mu, \xi)\left(\xi \sqcap \xi^{\sharp}\right) & & \{f \sqsubseteq \xi\} \\
& =\inf (\mu, \xi) . & & \left\{\xi \sqcap \xi^{\sharp} \sqsubseteq \operatorname{id}_{X}\right\}
\end{aligned}
$$

Since $f$ and $\inf (\mu, \xi)$ are functions, the identity $\inf (\mu, \xi)=\inf (\mu, \xi) f$ holds by Proposition $2.2(\mathrm{e})$. 
Remark 1. Suppose that the statement (c) in Proposition 3.3 is used as the definition of closure systems. Then an inclusion $\nu_{f}^{@} \Xi_{X}^{\sharp} \inf \left(\ni_{X}, \xi\right) \sqsubseteq \nu_{f}$ should be proved in the last Theorem 4.4. If $\left(\nu_{f}\right)^{@} \Xi_{X} \sharp \sqsubseteq \nabla_{I_{\wp}(X)}\left(\ni_{X} f\right)^{@}$ holds, then we have

$$
\begin{aligned}
\nu_{f}^{@} \Xi_{X} \sharp \inf \left(\ni_{X}, \xi\right) & \sqsubseteq \nabla_{I_{\wp}(X)}\left(\ni_{X} f\right)^{@} \inf \left(\ni_{X}, \xi\right) \\
& =\nabla_{I_{\wp}(X)} \inf \left(\left(\ni_{X} f\right)^{@} \ni_{X}, \xi\right) \\
& =\nabla_{I_{\wp}(X)} \inf \left(\ni_{X} f, \xi\right)
\end{aligned}
$$

and the proof will be completed after showing $\inf \left(\ni_{X} f, \xi\right) \sqsubseteq \inf \left(\ni_{X} f, \xi\right) f$ by the same proof in Theorem 4.4. The inclusion $\left(\nu_{f}\right)^{@} \Xi_{X} \sharp \sqsubseteq \nabla_{I_{\wp}(X)}\left(\ni_{X} f\right)^{@}$ is readily seen in $\operatorname{Rel}(S e t)$ to show the equivalence that $\mu \sqsubseteq \nabla_{V X} f$ iff $\mu \sqsubseteq \mu f^{\sharp} f$ by Dedekind formula (DC3) in Definition 2.1. However it is difficult (Curtis and Lowe (1995)) to show it without point wise discussion or point axioms. For we do not know how to internally manipulate Dedekind formula in power objects.

The following informal observation suggests how to construct a closure operation from a closure system:

$$
\begin{aligned}
\inf \{y \in \nu \mid x \leq y\} & =\inf (x \xi \sqcap \nu, \xi) \\
& =\inf \left(x \xi \sqcap x \nabla_{X I} \nu, \xi\right) \quad\left\{x \nabla_{X I}=\operatorname{id}_{I}\right\} \\
& =x \inf \left(\xi \sqcap \nabla_{X I} \nu, \xi\right) .
\end{aligned}
$$

TheOREM 4.5. Let $\xi: X \rightarrow X$ be a complete order. For all relations $\rho: I \rightarrow X$ the function $f_{\rho}=\inf \left(\xi \sqcap \nabla_{X I} \rho, \xi\right): X \rightarrow X$ is a closure operation.

Proof. Set $\hat{\rho}=\xi \sqcap \nabla_{X I} \rho$ for short. The antisymmetry and the completeness of $\xi$ guarantee that $f_{\rho}$ is a function (Cf. Proposition $2.2(\mathrm{p})$ ). The inclusion $f_{\rho} \sqsubseteq \xi$ directly follows from

$$
\begin{array}{rlll}
f_{\rho} & \sqsubseteq\left(\hat{\rho} \ominus \xi^{\sharp}\right) \ominus \xi & & \left\{f_{\rho}=\inf (\hat{\rho}, \xi)\right\} \\
& \sqsubseteq\left(\xi \ominus \xi^{\sharp}\right) \ominus \xi & & \{\hat{\rho} \sqsubseteq \xi\} \\
=\xi . & & \{\xi: \text { order }\}
\end{array}
$$

Next we remark that the identities $f_{\rho} \hat{\rho}=\xi \hat{\rho}=\hat{\rho}$ hold:

$$
f_{\rho} \hat{\rho} \sqsubseteq \xi \hat{\rho} \sqsubseteq \hat{\rho} \quad\left\{f_{\rho} \sqsubseteq \xi \text { and } \xi \xi \sqsubseteq \xi\right\}
$$

and

$$
\begin{array}{rlrl}
\hat{\rho} & \sqsubseteq\left[\left(\hat{\rho} \ominus \xi^{\sharp}\right) \ominus \xi\right] \sqcap \nabla_{X I} \rho & \{2.2(\mathrm{~g})\} \\
& \sqsubseteq\left(f_{\rho} \ominus \xi\right) \sqcap \nabla_{X I} \rho & & \left\{f_{\rho} \sqsubseteq \hat{\rho} \ominus \xi^{\sharp}\right\} \\
& =f_{\rho} \xi \sqcap \nabla_{X I} \rho & & \left\{f_{\rho}: \text { function and } 2.2(\mathrm{i})\right\} \\
& \sqsubseteq f_{\rho}\left(\xi \sqcap \nabla_{X I} \rho\right) . & & \{\text { DF }(\mathrm{DC} 3)\}
\end{array}
$$

Thus we have $f_{\rho} f_{\rho}=f_{\rho}$ from the following computation

$$
\begin{array}{rlrl}
f_{\rho} f_{\rho} & =f_{\rho} \inf (\hat{\rho}, \xi) & & \left\{f_{\rho}=\inf (\hat{\rho}, \xi)\right\} \\
& =\inf \left(f_{\rho} \hat{\rho}, \xi\right) & \left\{f_{\rho}: \text { function and } 2.2(\mathrm{o})\right\} \\
& =\inf (\hat{\rho}, \xi) & & \left\{f_{\rho} \hat{\rho}=\hat{\rho}\right\} \\
& =f_{\rho}, & &
\end{array}
$$


and $\xi \sqsubseteq f_{\rho} \xi f_{\rho}^{\sharp}$ from

$$
\begin{array}{rlll}
\xi^{\sharp} f_{\rho} & \sqsubseteq \xi^{\sharp}\left(\hat{\rho} \ominus \xi^{\sharp}\right) & & \left\{f_{\rho}=\inf (\hat{\rho}, \xi)\right\} \\
& =\xi^{\sharp}\left(\xi \hat{\rho} \ominus \xi^{\sharp}\right) & \{\hat{\rho}=\xi \hat{\rho}\} \\
& \sqsubseteq \hat{\rho} \ominus \xi^{\sharp} & & \left\{2.2(\mathrm{f}) \text { and } \alpha^{\sharp}(\alpha \ominus \beta) \sqsubseteq \beta\right\} \\
& =\inf (\hat{\rho}, \xi) \xi^{\sharp} & & \{2.2(\mathrm{n})\} \\
& =f_{\rho} \xi^{\sharp} . & &
\end{array}
$$

Finally we show the equivalence of closure systems and closure operations in Dedekind categories.

Theorem 4.6. Let $\xi: X \neg X$ be a complete order.

(a) If $f: X \rightarrow X$ is a closure operation, then $f=\inf \left(\xi \sqcap \nabla_{X X} f, \xi\right)=f_{\nu_{f}}$ holds.

(b) If $\nu: I \rightarrow X$ is a closure system, then $\nu=\nabla_{I X} \inf \left(\xi \sqcap \nabla_{X I} \nu, \xi\right)=\nu_{f_{\nu}}$ holds.

Proof. (a) Let $f: X \rightarrow X$ be a closure operation. First note that $\xi \sqcap \nabla_{X I} \nabla_{I X} f=$ $\xi \sqcap \nabla_{X X} f=f \xi f=\xi f$ follows from

$$
\begin{array}{rlll}
\xi \sqcap \nabla_{X X} f & \sqsubseteq\left(f \xi f^{\sharp} f^{\sharp} \sqcap \nabla_{X X}\right) f & \left\{\mathrm{DF} \text { and } \xi \sqsubseteq f \xi f^{\sharp}\right\} \\
& \sqsubseteq f \xi f & & \left\{f f=f \text { and } f^{\sharp} f \sqsubseteq \mathrm{id}_{X}\right\} \\
& \sqsubseteq \xi f & \{f \sqsubseteq \xi\} \\
& \sqsubseteq \xi \sqcap \nabla_{X X} f . & \{\xi \xi \sqsubseteq \xi\}
\end{array}
$$

Set $g=\inf \left(\xi \sqcap \nabla_{X X} f, \xi\right)$. Then it holds that

$$
\begin{aligned}
g & =\inf (f \xi f, \xi) & & \{\xi f=f \xi f\} \\
& =f \inf (\xi f, \xi) & & \{f: \text { function and } 2.2(\mathrm{o})\} \\
& =f g & & \\
& \sqsubseteq f \xi & & \{g \sqsubseteq \xi \text { by } 4.5\}
\end{aligned}
$$

and

$$
\begin{aligned}
g & \sqsubseteq g \xi^{\sharp} & & \left\{\operatorname{id}_{X} \sqsubseteq \xi\right\} \\
& =\xi f \ominus \xi^{\sharp} & & \{g=\inf (\xi f, \xi): \text { function and } 2.2(\mathrm{n})\} \\
& \sqsubseteq f \ominus \xi^{\sharp} & & \left\{\operatorname{id}_{X} \sqsubseteq \xi\right\} \\
& =f \xi^{\sharp} . & & \{f: \text { function }\}
\end{aligned}
$$

Hence we have

$$
g \sqsubseteq f \xi \sqcap f \xi^{\sharp}=f\left(\xi \sqcap \xi^{\sharp}\right) \sqsubseteq f,
$$

which proves $g=f$, because $g$ and $f$ are functions.

(b) First of all we note that $\nu \sqsubseteq \nabla_{I X} f_{\nu}$ follows from

$$
\begin{array}{rlll}
\nu & \sqsubseteq \nu\left(\operatorname{id}_{X} \sqcap \nabla_{X I} \nu\right) & & \{\mathrm{DF}\} \\
& \sqsubseteq \nu\left(\xi \sqcap \nabla_{X I} \nu \sqcap \xi^{\sharp}\right) & \left\{\operatorname{id}_{X} \sqsubseteq \xi \sqcap \xi^{\sharp}\right\} \\
& =\nu\left[\hat{\nu} \sqcap\left(\xi \Theta \xi^{\sharp}\right)\right] & & \left\{\xi^{\sharp}=\xi \Theta \xi^{\sharp}\right\} \\
& \sqsubseteq \nu\left[\hat{\nu} \sqcap\left(\hat{\nu} \ominus \xi^{\sharp}\right)\right] & & \{\hat{\nu} \sqsubseteq \xi\} \\
& \sqsubseteq \nu \inf (\hat{\nu}, \xi) & & \{2.2(\mathrm{~g})\} \\
& =\nu f_{\nu} . & &
\end{array}
$$


On the other hand

$$
\nabla_{I X} f_{\nu}=\nabla_{I X} \inf (\hat{\nu}, \xi) \sqsubseteq \nu
$$

holds because $\nu$ is a closure system and $\hat{\nu} \sqsubseteq \nabla_{X I} \nu$.

Remark 2. In the proof of Theorem 4.6 (b) the inclusion $\nabla_{I X} f_{\nu} \sqsubseteq \nu$ has been proved by virtue of Definition 3.4 of closure systems applying the statement (b) in Proposition 3.3. The first point axiom (PA1) might be needed to show the inclusion using the statement (a). In the concrete case (iv) in Introduction the containment $f_{S}(X) \subseteq S$ for a closure system $S$ readily follows from $f_{S}(x)=\inf S_{x} \in S$ obtained by the definition of (concrete) closure systems.

\section{Conclusion}

In this paper authors have studied the definitions of closure systems and closure operations in Dedekind categories. In particular we could prove the equivalence of closure systems and closure operations in Dedekind categories without point axioms by selecting a suitable definition of closure systems. This means that the equivalence also holds for not only (usual) set theory but higher-order intuitionistic set theory, since a Dedekind category with power objects may be regarded as the relation category of a topos. Also we encountered one concrete inclusion $\left(\nabla_{I X} f\right)^{@} \Xi_{X} \sharp \sqsubseteq \nabla_{I_{\wp}(X)}\left(\ni_{X} f\right)^{@}$ with a so-called traditional difficulty ,indicated by Curtis and Lowe (1995), to manipulate intermediate variables in relational methods. The idea given in the paper proposes a possible way to avoid such difficulties in relation algebras. The proof of the equivalence was rather technical than an ordinary proof with set theory. However the relational proofs might be similar to programs with functional languages and so will be useful in the future, for example, to analyse some topological structure of algebraic systems such as Boolean algebras in Dedekind categories by Kawahara (2008) .

Acknowlegdement The authors appreciate an anonymous referee for his helpful comments and suggestions.

\section{References}

Bergharmmer, R. and Neumann, F. (2005). RELVIEW - An OBDD-based computer algebra system for relations, Lecture Notes in Computer Science 3718. 40 - 51.

Curtis, S. and Lowe, G. (1995). A graphical calculus, Lecture Notes in Computer Science 947 . $214-231$

Freyd, P. and Scedrov, A. (1990). Categories, allegories, North-Holland, Amsterdam.

Ganter, B. and Wille, R. (1999). Formal Concept Analysis, Springer-Verlag, BerlinHeidelberg.

Ishida, T., Honda, K. and Kawahara, Y. (2008). Formal concepts in Dedekind categories, Lecture Notes in Computer Science 4988, 220 - 233.

Kawahara, Y. (2007). Urysohn's lemma in Schröder categories, Bull. Inform. Cybernet. 39, $69-81$. 
Kawahara, Y. (2008). Boolean algebras and Stone maps in Schröder categories, Lecture Notes in Computer Science 4988, 259 - 274.

Mac Lane, S. (1999). Categories for the working mathematician, Springer-Verlag.

Olivier, J-P. and Serrato, D. (1980). Catégories de Dedekind. Morphismes dans les Catégories de Schröder, C. R. Acad. Sci. Paris $260939-941$.

Schmidt, G. and Ströhlein, T. (1993). Relations and graphs - Discrete Mathematics for Computer Science -, pringer-Verlag, Berlin.

Schmidt, R. (Ed.) (2006). Relations and Kleene algebra in computer science, Lecture Notes in Computer Science $\mathbf{4 1 3 6 .}$

Tarski, A. (1941). On the calculus of relations, J. Symbolic Logic 6 73-89.

Received June 17, 2008

Revised August 8, 2008 